\title{
(2) OPEN ACCESS \\ Corneal biomechanics and biomechanically corrected intraocular pressure in primary open-angle glaucoma, ocular hypertension and controls
}

\author{
Riccardo Vinciguerra (1) ,' Salwah Rehman, ${ }^{2}$ Neeru A Vallabh, ${ }^{2}$ Mark Batterbury, ${ }^{2}$ \\ Gabriela Czanner, ${ }^{3}$ Anshoo Choudhary, ${ }^{2}$ Robert Cheeseman, ${ }^{2}$ Ahmed Elsheikh, ${ }_{1}$ \\ Colin E Willoughby ${ }^{5}$
}

'Birmingham and Midland Eye Centre, Sandwell and West Birmingham Hospitals, Birmingham, UK

${ }^{2}$ St Paul's Eye Unit, Royal Liverpool University Hospital, Liverpool, UK

${ }^{3}$ Department of Biostatistics, University of Liverpool, Liverpool, UK

${ }^{4}$ School of Engineering, University of Liverpool, Liverpool, UK

BBiomedical Sciences Research Institute, University of Ulster, Coleraine, UK

Correspondence to Dr Riccardo Vinciguerra, Birmingham and Midland Eye Centre, Birmingham B18 7JQ, UK; vinciguerra.riccardo@gmail. com

Received 5 November 2018 Revised 6 February 2019 Accepted 26 February 2019 Published Online First 28 March 2019

\section{Check for updates}

(C) Author(s) (or their employer(s)) 2020. Re-use permitted under CC BY-NC. No commercial re-use. See rights and permissions. Published by BMJ.

To cite: Vinciguerra $R$, Rehman S, Vallabh NA, et al. Br J Ophthalmol

2020;104:121-126.

\section{ABSTRACT}

Aims To compare the biomechanically corrected intraocular pressure (IOP) estimate (bIOP) provided by the Corvis-ST with Goldmann applanation tonometry (GAT-IOP) in patients with high-tension and normaltension primary open-angle glaucoma (POAG; HTG and NTG), ocular hypertension (OHT) and controls. Moreover, we compared dynamic corneal response parameters (DCRs) of the Corvis-ST in POAG, OHT and controls, evaluated the correlation between global visual field parameters mean deviation and pattern SD (MD and PSD) and DCRs in the POAG group.

Methods 156 eyes of 156 patients were included in this prospective, single-centre, observational study, namely 41 HTG and 33 NTG, 45 OHT cases and 37 controls. Central corneal thickness (CCT), GAT-IOP and bIOP were measured, GAT-IOP was also adjusted for CCT (GATAdj). DCRs provided by Corvis-ST were evaluated, MD and PSD were recorded by 24-2 full-threshold visual field. To evaluate the difference in DCRs between $\mathrm{OHT}$, HTG and NTG, a general linear model was used with sex, medications and group as fixed factors and bIOP and age as covariates.

Results There was a significant difference between GAT-IOP, GATAdj and bIOP in NTG and HTG, OHT and controls. NTG corneas were significantly softer and more deformable compared with controls, OHT and HTG as demonstrated by significantly lower values of stiffness parameters $\mathrm{A} 1$ and highest concavity and higher values of inverse concave radius (all $p<0.05$ ). There was a significant correlation $(p<0.05)$ between MD, PSD and many DCRs with POAG patients with softer or more compliant corneas more likely to show visual field defects.

Conclusions Corneal biomechanics might be a significant confounding factor for IOP measurement that should be considered in clinical decision-making. The abnormality of corneal biomechanics in NTG and the significant correlation with visual field parameters might suggest a new risk factor for the development or progression of NTG.

\section{INTRODUCTION}

Glaucoma is a disease characterised by progressive optic neuropathy and visual field loss with or without raised intraocular pressure (IOP). ${ }^{1}$ Normal-tension glaucoma (NTG) is a form of primary open-angle glaucoma (POAG), in which
IOP remains within normal levels; while hightension glaucoma (HTG) is associated with elevated IOP. Biomechanical properties of the optic nerve head $(\mathrm{ONH})$ and peripapillary scleral connective tissue have been postulated to determine how these structures respond to IOP, which may account for why some patients are susceptible to glaucomatous damage even under normal levels of IOP. $^{2}$ Nevertheless, since the cornea and sclera are continuous collagenous sheaths, made up of similar extracellular matrix constituents, an hypothesis could be that the biomechanical properties of the cornea may be somehow related to those of the lamina cribrosa (LC) or peripapillary sclera, which might determine the response of the ONH to IOP and the amount of axonal nerve damage. ${ }^{3}$ Assessment of corneal biomechanics may, therefore, offer an indirect measurement of the LC elasticity, and hence a possible indication of susceptibility to glaucomatous damage. However, it must be noted that the properties of the cornea are not constant from centre to periphery and through the sclera to the back of the eye. This link has been previously hypothesised in certain forms of glaucoma (NTG) which showed that abnormal corneal biomechanics was associated with progressive visual field loss. ${ }^{4}$ It is well known that the reduction of IOP is neuroprotective, in the sense that it delays or even prevents the structural and functional damage of optic nerve axons in glaucoma, including NTG. ${ }^{5-7}$ For this reason, the accurate measurement of IOP is an essential component of glaucoma management including case definition and in planning treatment. However, the accuracy of IOP measurement is influenced by the biomechanical properties of the cornea, of which the most important are corneal thickness and material stiffness. ${ }^{8}$ There have been numerous attempts using different devices to introduce an IOP estimate that would take into account corneal biomechanics. ${ }^{9-12}$

The challenge to produce IOP measurements with reduced biomechanical effect has also been addressed by the non-contact tonometer Corvis-ST (Oculus, Wetzlar, Germany) in the form of biomechanically corrected IOP (bIOP). The bIOP algorithm was developed using numerical, finite element simulations of the Corvis-ST procedure applied on human eye models with different topographies, thickness profiles, material properties and IOP values, and was shown to be significantly less affected by corneal parameters. ${ }^{13}$ Recently, the 
bIOP correction has been successful in providing close estimates of true IOP in ex vivo tests conducted on human donor eye globes, and in reducing association with the cornea's thickness. ${ }^{14}$

The aim of this study was to compare the bIOP estimates with Goldmann applanation tonometry IOP (GAT-IOP) in patients with POAG (HTG and NTG), ocular hypertension (OHT) and in healthy controls. Further, we aimed to assess and compare the dynamic corneal response parameters (DCRs), provided by the Corvis-ST, in the POAG, OHT and control groups, and evaluate the correlation between visual field parameters and corneal biomechanics in the POAG group.

\section{MATERIALS AND METHODS Population}

Patients diagnosed using the criteria described below with (POAG: HTG and NTG), OHT and healthy subjects were recruited over a period of 8 months in St Paul's Eye Unit, Royal Liverpool University Hospital, UK. All patients provided informed consent for using their anonymised data in the study prior to the study commencement.

\section{Inclusion criteria}

POAG was defined as open-angle gonioscopy, glaucomatous optic disc and an abnormal visual field (VF) consistent with glaucoma confirmed by at least two reliable VF examinations. The definition of glaucomatous visual field defect was defined by two glaucoma hemifield tests graded "outside normal limits" and a cluster of three contiguous points at the $5 \%$ level on the pattern deviation plot, using the threshold test strategy with the 24-2 test pattern of the Zeiss-Humphrey field analyzer. Patients were divided into HTG and NTG based on an untreated GAT-IOP greater or lower than $21 \mathrm{~mm} \mathrm{Hg}$. OHT patients were defined as normal VF with an untreated GAT-IOP greater than $21 \mathrm{~mm} \mathrm{Hg}$, respectively. Healthy controls had an untreated GAT-IOP lower than $21 \mathrm{~mm} \mathrm{Hg}$, healthy discs and no ocular pathologies.

\section{Exclusion criteria}

- Hypermetropia or myopia $>5$ diopters (D), and/or astigmatism $>3$ D, best-corrected visual acuity $<20 / 40$.

- Ocular conditions that could mimic glaucomatous visual field loss particularly congenital or acquired optic nerve diseases, or systemic conditions that could affect ocular blood flowparticularly diabetes mellitus and cerebrovascular diseases.

- Previous ocular or intraocular surgery or previous trauma or corneal scarring.

\section{Ophthalmological examination}

All participants underwent initial complete ophthalmic examination. The OHT and POAG groups also underwent automated perimetry using Humphrey Field Analyzer II (Carl Zeiss Meditec, Jena, Germany), with a full threshold 24-2 SITA standard programme. Global visual field parameters, including mean deviation (MD) and pattern SD (PSD), were recorded. IOP (mean of 3 measurements) and central corneal thickness (CCT) (mean of 25 measurements, one touch) were recorded using (GAT-IOP, Haag-Streit, Switzerland) and ultrasound pachymetry (DGH 55B Pachmate 2, DGH Technologies, Exton, Pennsylvania, USA), respectively, as well as corneal biomechanics and IOP measurement using the Corvis-ST. GAT-IOP was adjusted for pachymetry (GATAdj) using the manufacturer's correction algorithm provided with the Pachmate 2, which is based on a reference corneal thickness of $545 \mu \mathrm{m}$ from the work of Kohlhaas et al ${ }^{11}$

\section{Corvis measurements}

All measurements with the Corvis-ST (software V.6.08r22) were captured by automatic release on alignment with the corneal apex and were all with 'OK' quality score. The DCRs used in the study are summarised in online supplementary table $1^{15} 16$ and were previously described. ${ }^{17} 18$ We included inverse concave radius, stiffness parameters (SP-A1) (referenced at first applanation) and SP-HC (referenced at highest concavity) and deformation amplitude ratio (DA ratio) as they were shown in earlier studies to be well correlated with corneal biomechanics and relatively independent of IOP. ${ }^{18} 19$ Together with DCRs, bIOP estimate $^{1314}$ was recorded.

\section{Statistical analysis}

Only one eye per patient (when both eyes could be included) was randomly selected and included in the analysis. Statistical analyses were performed using the SPSS software (V.24, IBM). Sample size calculation was done using G Power V.3.1.9.3 software, assuming clinically relevant effect size of 0.4 for the difference in SP-A1, also suggested by previous unpublished study. With four groups, five covariates, alpha of 0.05 and power of 0.85 , assuming general linear model (GLM) the calculated sample size was of minimum 131 subjects. Descriptive statistics were calculated for the DCR parameters, as well as GATIOP, GATAdj and bIOP. Differences between the groups (OHT, HTG, NTG and normal) were evaluated with analysis of variance and Bonferroni post hoc test or logistic regression when appropriate. Additionally, to evaluate the difference in corneal biomechanics between OHT, HTG and NTG, a GLM was used with sex, medications and group as fixed factors and bIOP and age as covariates. Medications were defined as four categorical variables each having two levels (present/absent). Additionally, to exclude CCT as a confounding factor, a subgroup of OHT, NTG and HTG patients were matched with CCT (maximum difference $8 \mu \mathrm{m}$ ) and by medication (all of them only on prostaglandin analogues). A further GLM with SP-A1 as dependent variable was performed with group as fixed factor and bIOP and age as covariates. The association between the DCR parameters and visual field indices such as MD and pattern SD (PSD) was expressed with Spearman correlation coefficient. A $p<0.05$ was considered statistically significant.

\section{RESULTS}

Patient demographics and IOPs are summarised in table 1.

Table 1 Demographics and ocular characteristics for patients with ocular hypertension (OHT), primary open-angle glaucoma hightension glaucoma (HTG), normal-tension glaucoma (NTG) and healthy eye controls, mean \pm SD

\begin{tabular}{llllll}
\hline & Normal & OHT & HTG & NTG & P value \\
\hline No of eyes & 37 & 45 & 41 & 33 & NA \\
Eye (right/left) & $22 / 15$ & $25 / 15$ & $20 / 16$ & $12 / 18$ & 0.619 \\
Sex (male/female) & $10 / 27$ & $18 / 22$ & $20 / 16$ & $15 / 15$ & 0.003 \\
CCT $(\mu \mathrm{m})$ & $553 \pm 33$ & $547 \pm 53$ & $522 \pm 31$ & $504 \pm 34$ & $<0.001$ \\
Age (years) & $71.8 \pm 10.3$ & $60 \pm 12.0$ & $70.9 \pm 10.3$ & $75.1 \pm 9.5$ & $<0.001$ \\
\hline bIOP $(\mathrm{mm} \mathrm{Hg})$ & $13.4 \pm 2.8$ & $17.0 \pm 4.1$ & $14.8 \pm 3.1$ & $12.9 \pm 2.3$ & $<0.001$ \\
GAT-IOP $(\mathrm{mm} \mathrm{Hg})$ & $16.4 \pm 2.4$ & $22.1 \pm 4.8$ & $17.2 \pm 4.9$ & $13.7 \pm 1.8$ & $<0.001$ \\
GATAdj $(\mathrm{mm} \mathrm{Hg})$ & $15.3 \pm 2.7$ & $20.8 \pm 5.5$ & $18.6 \pm 4.3$ & $16.3 \pm 2.7$ & $<0.001$ \\
\hline
\end{tabular}

CCT, central corneal thickness; GATAdj, Goldmann applanation tonometry intraocular pressure adjusted for pachymetry; GAT-IOP, Intraocular pressure measured by Goldmann applanation tonometry; NA, not applicable; bIOP, biomechanically-corrected intraocular pressure estimate provided by the Corvis ST. 
Table 2 Showing the number and percentage of patients on glaucoma medication and mean values \pm SD of mean deviation (MD) and pattern SD (PSD) of patients in the groups of ocular hypertension (OHT), high-tensions glaucoma (HTG) and normal-tension glaucoma (NTG)

\begin{tabular}{llllc}
\hline & OHT & HTG & NTG & P value \\
\hline Prostaglandin analogues & $31(58.5 \%)$ & $37(78 \%)$ & $23(69.7 \%)$ & 0.260 \\
$\beta$-blockers & $7(13.2 \%)$ & $20(42.5 \%)$ & $11(30.5 \%)$ & 0.058 \\
CA-inh & $5(9.4 \%)$ & $17(36.1 \%)$ & $10(27.7 \%)$ & 0.053 \\
$\alpha$ agonist & $3(5.7 \%)$ & $4(8.5 \%)$ & $1(2.8 \%)$ & 0.837 \\
Mean Deviation & $-1.46 \pm 1.90$ & $-7.60 \pm 8.45$ & $-6.79 \pm 6.28$ & $<0.001$ \\
Post hoc $p$ value & $<0.001$ & 1.000 & & \\
Pattern Standard & $1.99 \pm 1.36$ & $8.40 \pm 10.75$ & $6.58 \pm 3.88$ & $<0.001$ \\
Deviation & & & & \\
Post hoc $p$ value & $<0.001$ & 0.199 & & \\
\hline
\end{tabular}

$P$ values express the difference between the groups. When applicable post hoc $p$ values are between NTG and the other groups.

CA-inh, carbonic anhydrase inhibitors.

The mean CCT in NTG was significantly lower than in normal $(\mathrm{p}<0.001)$ and OHT $(\mathrm{p}=0.004)$ groups, but similar to HTG $(\mathrm{p}=1.0)$. Table 2 shows the number and percentage of patients under each type of topical glaucoma medications.

There was no significant difference between the groups (OHT, NTG and HTG) in terms of any of the medication, however, the $\mathrm{p}$ values of carbonic anhydrase inhibitors and beta-blockers approached significance (table 2).

\section{Intraocular pressure}

Comparative analysis showed a significant difference between the values of GAT-IOP, GATAdj and bIOP among the groups and within the groups $(\mathrm{p}<0.001$, figure 1$)$.

\section{Within the groups}

The main result of this analysis was the significant difference between the values of GAT-IOP, GATAdj and bIOP in all groups $(\mathrm{p}<0.001)$. In controls and OHT, the mean values of GAT-IOP were significantly higher than GATAdj and bIOP, equally GATAdj was significantly higher than bIOP $(\mathrm{p}<0.01)$.

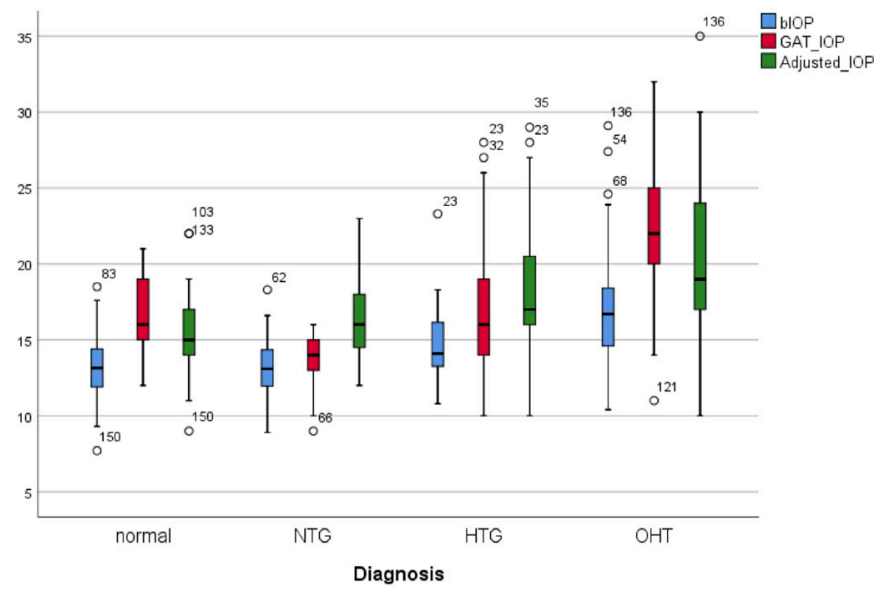

Figure 1 Box and whiskers plot for bIOP, Goldmann applanation tonometry IOP (GAT) and Goldmann adjusted (GATAdj) in ocular hypertension (OHT), primary open-angle glaucoma high-tension glaucoma (HTG), normal-tension glaucoma (NTG) and healthy eye controls. bIOP, biomechanically corrected intraocular pressure; IOP, intraocular pressure.
Conversely, GATAdj was significantly higher than GAT-IOP in HTG and NTG groups, and GAT-IOP was significantly higher than bIOP $(\mathrm{p}<0.01)$.

\section{Between the groups}

\section{GAT-IOP between the groups}

The Bonferroni post hoc tests showed that mean GAT-IOP in OHT group was significantly higher than in all other groups $(\mathrm{p}<0.001)$. Similarly, HTG POAG patients showed higher values of GAT-IOP compared with NTG $(p=0.003)$ and lower than OHT $(p<0.001)$ but very similar to normal $(p=1.00)$. The values of GAT-IOP of OHT were on average $5.6 \mathrm{~mm} \mathrm{Hg}$ higher than controls.

\section{GATAdj-IOP between the groups}

The comparative analysis of GATAdj pressure between the groups showed that the mean values of OHT and HTG with GATAdj were significantly higher compared with controls $(\mathrm{p}<0.001$ and $\mathrm{p}=0.009$, table 1$)$.

\section{bIOP between the groups}

The results for bIOP showed no difference between the values in POAG (HTG and NTG) and control groups but a significant difference with OHT. The mean difference between OHT and normal was $3.6 \mathrm{~mm} \mathrm{Hg}(\mathrm{p}<0.001)$.

\section{Corneal biomechanics}

The analysis of the Corvis DCR parameters, performed with GLM which took into account age, sex, bIOP and medication, showed a significant difference between at least one group and the others in all evaluated parameters (table 3 ).

The main result of this subanalysis is the evidence that NTG corneas are more compliant and deformable under the air puff compared with controls, OHT and HTG patients as showed by significantly lower values of SP-A1 and HC and significant higher values of inverse concave radius and $\mathrm{DA}$ ratio (figure $2 \mathrm{~A}-\mathrm{D}$ ). A subgroup of OHT (11), NTG (11) and HTG (13) patients were matched with CCT and by medication (all of them only on prostaglandin analogues). The comparative analysis with GLM confirmed the previous results, demonstrating that NTG corneas are softer and more deformable under the air puff (lower values of SP-A1) compared with OHT and HTG patients $(\mathrm{p}=0.017$ overall, NTG vs OHT $p=0.023$, NTG vs HTG $p=0.007$ ) even when matching with CCT.

\section{Visual field and corneal biomechanics}

The visual field indices of POAG (HTG and NTG) and OHT patients are summarised in table 2. The correlation analysis between corneal biomechanics, MD and PSD included only POAG patients (HTG and NTG). The main result of this subanalysis is the evidence that POAG patients with softer and more deformable corneas are significantly more likely to show visual field defects than stiffer ones. This was demonstrated by a significant negative correlation between $\mathrm{MD}$ and $\mathrm{DA}$ ratio $(\mathrm{cc}=-0.261 \mathrm{p}=0.018)$, inverse concave radius $(\mathrm{cc}=-0.242$, $\mathrm{p}=0.028)$ and a significant positive correlation with SP-A1 $(\mathrm{cc}=0.279, \mathrm{p}=0.011)$ and SP-HC $(\mathrm{cc}=0.240, \mathrm{p}=0.030)$.

Similarly, the correlation analysis of corneal biomechanics and PSD showed a significant positive correlation with DA ratio $(\mathrm{cc}=0.299, \mathrm{p}=0.006)$, inverse concave radius $(\mathrm{cc}=0.305$, $\mathrm{p}=0.005)$ and a significant negative correlation with SP-A1 $(\mathrm{cc}=-0.346, \mathrm{p}=0.001)$ and SP-HC $(\mathrm{cc}=-0.329, \mathrm{p}=0.003)$. 
Table 3 Showing mean values \pm SD of inverse radius, deformation amplitude ratio (DA ratio), stiffness parameter applanation 1 (SP-A1) and SP at highest concavity (SP-HC) in ocular hypertension (OHT), high-tensions glaucoma (HTG), normal-tension glaucoma (NTG) and controls

\begin{tabular}{|c|c|c|c|c|c|}
\hline & Normal & OHT & HTG & NTG & Overall $p$ value \\
\hline Inverse radius & $8.32 \pm 1.02$ & $8.09 \pm 2.17$ & $8.54 \pm 1.05$ & $9.39 \pm 1.01$ & 0.034 \\
\hline Post hoc $p$ value NTG versus other groups & 0.016 & 0.001 & 0.087 & & \\
\hline Post hoc $p$ value HTG versus other groups & 0.669 & 0.049 & & 0.258 & \\
\hline DA ratio & $1.57 \pm 0.05$ & $1.57 \pm 0.05$ & $1.61 \pm 0.05$ & $1.63 \pm 0.04$ & 0.015 \\
\hline Post hoc $p$ value NTG versus other groups & 0.057 & 0.028 & 1.0000 & & \\
\hline Post hoc $p$ value HTG versus other groups & 0.242 & 0.050 & & 0.132 & \\
\hline SP-A1 & $108.5 \pm 16.1$ & $117.6 \pm 25.4$ & $104.0 \pm 18.8$ & $88.1 \pm 15.2$ & $<0.001$ \\
\hline Post hoc $p$ value NTG versus other & $<0.0001$ & $<0.0001$ & 0.005 & & \\
\hline Post hoc $p$ value HTG versus other & 0.234 & 0.076 & & 0.003 & \\
\hline SP-HC & $5.73 \pm 2.80$ & $8.49 \pm 3.43$ & $5.63 \pm 3.05$ & $3.17 \pm 1.98$ & 0.004 \\
\hline Post hoc $p$ value NTG versus other & 0.005 & $<0.0001$ & 0.010 & & \\
\hline Post hoc $p$ value HTG versus other & 0.150 & 0.071 & & 0.017 & \\
\hline
\end{tabular}

P values of general linear model. Post hoc $p$ values are between NTG or HTG and the other groups.

\section{DISCUSSION}

Glaucoma is a complex disease and clinically challenging due to its multifactorial aetiology. ${ }^{20}$ Currently, IOP is the only modifiable risk factor and the current gold standard for IOP measurement is the Goldmann applanation tonometry (GAT-IOP). However, the accuracy of GAT-IOP is influenced by overall corneal stiffness which varies with corneal thickness and material behaviour. ${ }^{8}$ The effect of corneal material properties, in particular the mechanical stiffness, on GAT-IOP is expected to be considerable. $^{8} 21$

This study aimed to evaluate and compare the bIOP algorithm with the GAT-IOP in patients with primary open angle glaucoma (POAG: NTG and HTG), ocular hypertension (OHT) and controls. Subsequently, we aimed to assess and compare corneal biomechanics in the described groups and if those biomechanical factors were correlated with the visual field defect.

\section{IOP results}

The comparative analysis showed a significant difference between the values of GAT-IOP, GATAdj and bIOP in POAG (HTG and NTG), OHT and controls. In all the groups, bIOP was significantly lower than GAT and GATAdj. In previous studies, normal subjects mean GAT and bIOP values were very similar ${ }^{1822}$ and a recent study demonstrated that bIOP equates to manometric IOP measurements in ex vivo eyes. ${ }^{14}$ The difference between GAT and bIOP in our study must, therefore, relate to the 'adjusted factors' that bIOP corrects for, namely, age, CCT and corneal biomechanics, or by the higher accuracy and repeatability of Corvis-ST bIOP measurements compared with GAT. ${ }^{23}$ As a matter of fact in the study which described the normality values of bIOP the mean age was $38 \pm 16$ years, mean CCT was $543 \pm 33 \mu \mathrm{m}$ and mean DA ratio was ranging from 1.452 to $1.727 .{ }^{18}$ These values are different compared with the normal population evaluated in the present study that has a higher age and slightly thicker corneas which would cause an overestimation of GAT.

Previous studies showed that GAT-IOP is affected by a margin that varied between 0.7 and $7.1 \mathrm{~mm} \mathrm{Hg}$ per each $100 \mu \mathrm{m}$ change in CCT. ${ }^{24} 25$ The significance of this error margin should be evaluated critically given that the progression risk in patients with diagnosed glaucoma is reported to be increased between $10 \%$ and $12 \%$ for each $1 \mathrm{~mm} \mathrm{Hg}$ increase in $\mathrm{IOP}^{26}$ and hence the consequence of this error could produce significant numbers of false-positives and false-negatives in glaucoma risk profiling.

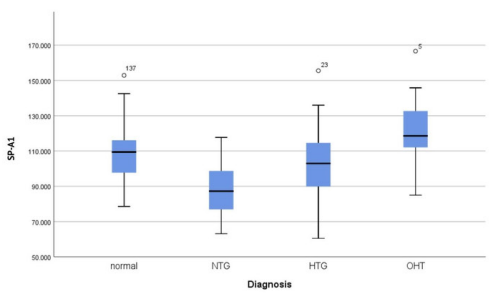

C

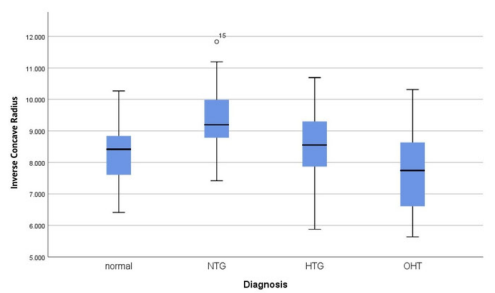

B

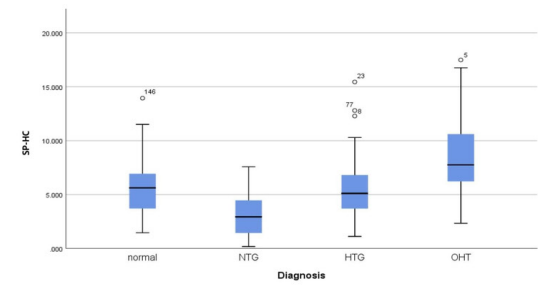

D

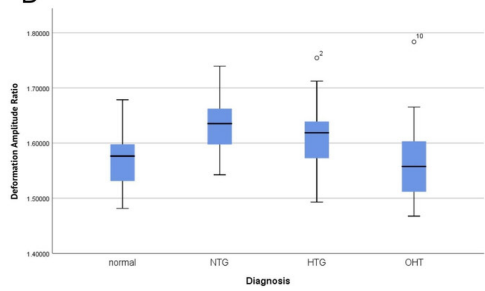

Figure 2 (A) Box and whiskers plot for stiffness parameter A1, (B) stiffness parameter highest concavity, (C) inverse concave radius, (D) deformation amplitude ratio in ocular hypertension (OHT), primary open-angle glaucoma high-tension glaucoma (HTG), normal-tension glaucoma (NTG) and healthy eye controls. 
Moreover, a previous report showed that differences in corneal biomechanics across individuals may have greater impact on IOP measurement errors than corneal thickness or curvature. ${ }^{8}$ This result underlines the importance of using an IOP estimate that is not affected by material properties, age and thickness. Furthermore, it should be noted that in controls and OHT, GAT was shown to be higher than GATAdj, while in both glaucoma groups, it was the opposite. This would suggest that the relative differences change with disease, such that the adjustment for CCT no longer seems to work. This is consistent with predictions of the work of Liu and Roberts, where stiffer corneas have a stronger relationship between CCT and IOP measurement error, while in softer corneas, this relationship is diminished. ${ }^{8}$

In OHT patients, GAT-IOP was on average higher than 21 $\mathrm{mm} \mathrm{Hg}$ while bIOP was lower. This result suggests that with the use of bIOP, OHT patients (who are patients that, even with high IOP did not yet progress to glaucoma) present an IOP that is within normal limits $(<21 \mathrm{~mm} \mathrm{Hg})$ whereas with GAT the IOP is higher than the normal range. Previous formulas were proposed to improve the management of OHT such as in the OHT treatment study, however, the definition of OHT changed depending on which adjustment formula was used. ${ }^{27}$ Corvis bIOP has many advantages when compared with GAT and GATAdj, in particular it is non-contact, it does not require anaesthesia, it is developed with numerical modelling and is able to correct for age and corneal biomechanics as well as CCT. ${ }^{13}$

A better estimate of IOP (such as bIOP) could help to separate OHT patients at high risk of progression to glaucoma from those individuals that are only overestimated by standard tonometers, thus improving glaucoma diagnosis and reducing public health costs. Further studies are needed to evaluate if bIOP would be able to provide a better way to define OHT or follow-up patients with glaucoma.

\section{Corneal biomechanics Results}

The main result of this subanalysis (which took age, bIOP and medications into account) is the evidence that NTG corneas are softer and more deformable under the air puff compared with controls, OHT and HTG. This was demonstrated by significantly lower values of SP-A1 and HC and significant higher values of inverse concave radius and DA ratio. A softer cornea, in general, would be more deformed by the air puff, which will be identified by the Corvis with a higher inverse radius of concavity and DA ratio. Similarly, it will show lower values of the (SP-A1 and SP-HC) which are two parameters correlated with overall stiffness. ${ }^{28}$

CCT was purposely not added into the model because it does not meet all the criteria of confounding ${ }^{29}$ and hence would lead to a bias. Finally, our last subanalysis which evaluated OHT, NTG and HTG patients that were matched with CCT and medication also confirmed the outcomes of the main analysis and excluded the possibility that the results would be driven by the difference in CCT.

Based on the evidence that NTGs have corneas that are softer and more deformable, the evaluation of corneal biomechanics might help in the management/risk stratification of glaucomatous patients.

In the present, the results shown refer to overall stiffness (geometrical stiffness and material stiffness together); and show that NTGs behave softer compared with other groups. Nevertheless, we cannot prove that the material stiffness of NTG patients is either softer or stiffer compared with the other groups, more studies will be needed, possibly with the use of new indexes not influenced by corneal geometry, to evaluate this aspect. Our study shows significantly softer corneas (more deformable) in NTG patients compared with controls, OHT and HTG with new DCRs of the Corvis-ST that are relatively independent from IOP. ${ }^{18}$ Although previous studies focused mainly on HTG showed less deformable corneas, ${ }^{30} 31$ older DCRs known to be influenced by IOP were used..$^{18}$ There is a plethora of studies with Ocular Response Analyzer (ORA) in NTG and POAG; however, those findings are not directly comparable to the ones of the Corvis for two reasons. First, the ORA has a variable magnitude air puff, and biomechanical response is dependent on load. ${ }^{15}$ Second, corneal hysteresis is a viscoelastic term and does not directly correlate to stiffness. Previous literature found that NTG had lower $\mathrm{CH}$ compared with POAG and OHT, but this cannot be interpreted as either a stiffer or a softer cornea. ${ }^{32}$ Low hysteresis is found in keratoconus with a soft cornea, as well as with higher IOP, which is a stiffer cornea. ${ }^{33}$

\section{Correlation between visual field defect and corneal biomechanics}

The main finding of this subanalysis is that HTG and NTG patients with corneas that are more deformed by the air puff (softer) are significantly more likely to show visual field defects than those with stiffer corneas (less deformed). These findings are partly in agreement (since we did not evaluate progression) with previous literature that showed that DCRs are related to the progression of $\mathrm{HTG}^{34}$ and NTG. ${ }^{35}$ As previously mentioned, results of the DCRs of the Corvis-ST should not be compared with those ORA as not equivalent.

In conclusion, we found a significant difference between Goldmann, Goldmann CCT adjusted and bIOP which should be related to the compensating factors that bIOP corrects for, namely, age, CCT and corneal biomechanics. NTG corneas are significantly softer compared with controls, OHT and HTG. Patients with glaucoma with more compliant corneas were more likely to show visual field defects.

Contributors RV initiated the project, designed data collection tools, monitored data collection for the whole trial, wrote the statistical analysis plan, cleaned and analysed the data, drafted and revised the paper. SR and NV collected and analysed the data as well as drafted and revised the paper. GC wrote the statistical analysis plan and revised the draft and approved the final version of the paper. $A E, C E W, M B, A C$ and $R C$ contributed substantially in conception and design of the study revised and approved the draft and the final version of the paper.

Funding The authors have not declared a specific grant for this research from any funding agency in the public, commercial or not-for-profit sectors.

Disclaimer OCULUS Optikgeräte GmbH did not take part in design, analysis or interpretation of the results.

Competing interests RV and AE are consultants for OCULUS Optikgeräte GmbH. Patient consent for publication Not required.

Ethics approval The study data were acquired under ethical approval of the Royal Liverpool University Hospital for service development audit purposes and adhered to the tenets of the Declaration of Helsinki.

Provenance and peer review Not commissioned; externally peer reviewed.

Open access This is an open access article distributed in accordance with the Creative Commons Attribution Non Commercial (CC BY-NC 4.0) license, which permits others to distribute, remix, adapt, build upon this work non-commercially, and license their derivative works on different terms, provided the original work is properly cited, appropriate credit is given, any changes made indicated, and the use is non-commercial. See: http://creativecommons.org/licenses/by-nc/4.0/.

ORCID ID

Riccardo Vinciguerra http://orcid.org/0000-0002-2280-9981 


\section{REFERENCES}

1 Tham Y-C, Li X, Wong TY, et al. Global prevalence of glaucoma and projections of glaucoma burden through 2040: a systematic review and meta-analysis. Ophthalmology 2014;121:2081-90.

2 Burgoyne CF, Downs JC, Bellezza AJ, et al. The optic nerve head as a biomechanical structure: a new paradigm for understanding the role of IOP-related stress and strain in the pathophysiology of glaucomatous optic nerve head damage. Prog Retin Eye Res 2005;24:39-73.

3 Helmy H, Leila M, Zaki AA. Corneal biomechanics in asymmetrical normal-tension glaucoma. Clin Ophthalmol 2016;10:503-10.

4 Spörl E, Terai N, Haustein M, et al. [Biomechanical condition of the cornea as a new indicator for pathological and structural changes]. Ophthalmologe 2009;106:512-20.

5 Kass MA, Heuer DK, Higginbotham EJ, et al. The ocular hypertension Treatment study: a randomized trial determines that topical ocular hypotensive medication delays or prevents the onset of primary open-angle glaucoma. Arch Ophthalmol 2002;120:701-13. discussion 829-30.

6 Leske MC, Heijl A, Hyman L, et al. Early manifest glaucoma trial: design and baseline data. Ophthalmology 1999;106:2144-53.

7 Miglior S, Zeyen T, Pfeiffer N, et al. Results of the European glaucoma prevention study. Ophthalmology 2005;112:366-75.

8 Liu J, Roberts CJ. Influence of corneal biomechanical properties on intraocular pressure measurement: quantitative analysis. J Cataract Refract Surg 2005;31:146-55.

9 Kanngiesser HE, Kniestedt C, Robert YCA. Dynamic contour tonometry: presentation of a new tonometer. J Glaucoma 2005;14:344-50.

10 Montard R, Kopito R, Touzeau 0, et al. [Ocular response analyzer: feasibility study and correlation with normal eyes]. J Fr Ophtalmol 2007:30:978-84.

11 Kohlhaas M, Boehm AG, Spoerl E, et al. Effect of central corneal thickness, corneal curvature, and axial length on applanation tonometry. Arch Ophthalmol 2006;124:471-6.

12 Elsheikh A, Alhasso D, Gunvant P, et al. Multiparameter correction equation for Goldmann applanation tonometry. Optom Vis Sci 2011;88:E102-12.

13 Joda AA, Shervin MMS, Kook D, et al. Development and validation of a correction equation for Corvis tonometry. Comput Methods Biomech Biomed Engin 2016;19:943-53.

14 Eliasy A, Chen K-J, Vinciguerra R, et al. Ex-vivo experimental validation of biomechanically-corrected intraocular pressure measurements on human eyes using the CorVis ST. Exp Eye Res 2018;175:98-102.

15 Roberts CJ. Concepts and misconceptions in corneal biomechanics. J Cataract Refract Surg 2014;40:862-9.

16 Ambrosio R, Jr, Ramos I, Luz A, et al. Dynamic Ultra-High Speed Scheimpflug Imaging for assessing corneal biomechanical properties. Rev Bras Oftalmol 2013;72.

17 Vinciguerra R, Ambrósio R, Elsheikh A, et al. Detection of keratoconus with a new biomechanical index. J Refract Surg 2016;32:803-10.

18 Vinciguerra R, Elsheikh A, Roberts CJ, et al. Influence of pachymetry and intraocular pressure on dynamic corneal response parameters in healthy patients. J Refract Surg 2016;32:550-61.
19 Roberts CJ, Mahmoud AM, Bons JP, et al. Introduction of two novel stiffness parameters and interpretation of air Puff-Induced biomechanical deformation parameters with a dynamic scheimpflug analyzer. J Refract Surg 2017;33:266-73.

20 Weinreb RN, Aung T, Medeiros FA. The pathophysiology and treatment of glaucoma: a review. JAMA 2014;311:1901-11.

21 Hamilton KE, Pye DC. Young's modulus in normal corneas and the effect on applanation tonometry. Optom Vis Sci 2008;85:445-50.

22 Yilmaz I, Altan C, Aygit ED, et al. Comparison of three methods of tonometry in normal subjects: Goldmann applanation tonometer, non-contact airpuff tonometer, and Tono-Pen XL. Clin Ophthalmol 2014;8:1069-74.

23 Lopes BT, Roberts CJ, Elsheikh A, et al. Repeatability and reproducibility of intraocular pressure and dynamic corneal response parameters assessed by the Corvis ST. J Ophthalmol 2017;2017:1-4.

24 Tonnu P-A, Ho T, Newson T, et al. The influence of central corneal thickness and age on intraocular pressure measured by pneumotonometry, non-contact tonometry, the Tono-Pen XL, and Goldmann applanation tonometry. Br J Ophthalmol 2005;89:851-4.

25 Gunvant P, Baskaran M, Vijaya L, et al. Effect of corneal parameters on measurements using the pulsatile ocular blood flow tonograph and Goldmann applanation tonometer. Br J Ophthalmol 2004;88:518-22.

26 Leske MC, Heijl A, Hussein M, et al. Factors for glaucoma progression and the effect of treatment: the early manifest glaucoma trial. Arch Ophthalmol 2003;121:48-56.

27 Brandt JD, Gordon MO, Gao F, et al. Adjusting intraocular pressure for central corneal thickness does not improve prediction models for primary open-angle glaucoma. Ophthalmology 2012;119:437-42.

28 Roberts CJ, Mahmoud AM, Bons JP, et al. Introduction of two novel stiffness parameters and interpretation of air Puff-Induced biomechanical deformation parameters with a dynamic scheimpflug analyzer. J Refract Surg. In Press 2017;33:266-73

29 VanderWeele TJ, Shpitser I. On the definition of a confounder. Ann Stat 2013;41:196-220

30 Lee R, Chang RT, Wong IYH, et al. Novel parameter of corneal biomechanics that differentiate normals from glaucoma. J Glaucoma 2016;25:e603-9.

31 Tian L, Wang D, Wu Y, et al. Corneal biomechanical characteristics measured by the CorVis scheimpflug technology in eyes with primary open-angle glaucoma and normal eyes. Acta Ophthalmol 2016;94:e317-24.

32 Shah S, Laiquzzaman M, Mantry S, et al. Ocular response analyser to assess hysteresis and corneal resistance factor in low tension, open angle glaucoma and ocular hypertension. Clin Exp Ophthalmol 2008;36:508-13.

33 Congdon NG, Broman AT, Bandeen-Roche K, et al. Central corneal thickness and corneal hysteresis associated with glaucoma damage. Am J Ophthalmol 2006;141:868-75.

34 Matsuura M, Hirasawa K, Murata H, et al. Using CorvisST tonometry to assess glaucoma progression. PLoS One 2017;12:e0176380.

35 Li BB, Cai Y, Pan YZ, et al. [The association between corneal biomechanical parameters and visual field progression in patients with normal tension glaucoma]. Zhonghua Yan Ke Za Zhi 2018;54:171-6. 